\title{
Spin transmission control in helical magnetic fields
}

\author{
Henri Saarikoski, ${ }^{*}$ Tobias Dollinger, and Klaus Richter \\ Department of Theoretical Physics, Regensburg University, 93040 Regensburg, Germany
}

(Received 23 July 2012; published 3 October 2012)

\begin{abstract}
We calculate spin transport in two-dimensional waveguides in the presence of spatially modulated Zeeman-split energy bands. We show that in a regime where the spin evolution is predominantly adiabatic the spin backscattering rate can be tuned via diabatic Landau-Zener transitions between the spin-split bands [Betthausen $e t$ al., Science 337, 324 (2012)]. This mechanism is tolerant against spin-independent scattering processes. Completely spinpolarized systems show full spin backscattering, and thus current switching. In partially spin-polarized systems a spatial sequence of Landau-Zener transition points enhances the resistance modulation via reoccupation of backscattered spin-polarized transport modes. We discuss a possible application as a spin transistor.
\end{abstract}

DOI: 10.1103/PhysRevB.86.165407

PACS number(s): 71.70.Ej, 72.25.Dc, 85.75.Hh

\section{INTRODUCTION}

Current technologies in semiconductor electronics have fundamental limits on the transistor switching times that can be achieved with low energy consumption. Integration of electron spin-based functionalities into devices may lead to faster operation. ${ }^{1}$ Datta and Das proposed an idea to modulate current in a spin transistor device via spin precession in a spin-orbit field. ${ }^{2}$ In their concept spin is injected from a ferromagnetic source into a channel of a two-dimensional electron gas (2DEG) where spin precesses in a gate-controlled spin-orbit field. The drain is another ferromagnet where spin magnetic moment orientation parallel (antiparallel) to the magnetization direction of the drain corresponds to the transistor "on" ("off") position. Signatures of the Datta-Das spin transistor mechanism have been demonstrated in a nonlocal measurement. ${ }^{3}$ However, signal levels are small due to issues concerning spin injection efficiency and fast spin relaxation. ${ }^{4}$ Fast spin decay makes information encoded in spin very volatile and limits its transmission range.

Recently an alternative way to achieve spin transistor action has been proposed: Stability of spin is enhanced by keeping spin transport in the adiabatic regime ${ }^{5}$ and spin transmission can then be controlled via Landau-Zener transitions in spatially modulated spin-split bands. ${ }^{6}$ This leads effectively to a tunable backscattering of spins which changes conductance and the degree of spin polarization of transmitted electrons in the device. The validity of this approach was shown in transport experiments ${ }^{6}$ in magnetically modulated diluted $(\mathrm{Cd}, \mathrm{Mn}) \mathrm{Te}$ magnetic semiconductor quantum wells where the $s$ - $d$ exchange interaction between electronic states and the localized magnetic moments of the Mn atoms gives rise to an enhanced $g$ factor and a giant Zeeman splitting. ${ }^{7}$ In the low-field limit at low temperatures the $g$ factor is approximately constant with values ranging up to several hundreds. In these experiments spin transistor action was realized by combining helical and tunable homogeneous magnetic field components. The helical field component was created by placing a premagnetized ferromagnetic stripe grating above the sample surface. A dysprosium stripe grating induces a stray field which is approximately helical in the plane of the 2DEG with a field strength of the order of $50 \mathrm{mT}$. Due to the giant $g$ factor the spin polarization of the ground state in this field was about $10 \%$. The giant Zeeman coupling is more than an order of magnitude larger than the Rashba and the Dresselhaus spin-orbit couplings in these magnetic fields. ${ }^{6}$

Motivated by these experiments, here we consider spin transmission control in helical magnetic fields in the presence of spin-independent disorder scattering and magnetic field coupling to orbital dynamics. We calculate spin transport in a two-terminal model device, sketched in Fig. 1. We show that current switching can be attained in fully spin-polarized systems despite disorder. This finding is in contrast to the Datta-Das spin transistor; its operation is disrupted if the mean free path of electrons is of the order of the channel length. We further show that transitions between transport modes induced by orbital coupling, which was not considered in Ref. 6, may enhance the resistance modulation in partially polarized waveguides.

\section{MODEL}

\section{A. Effective mass Hamiltonian}

We use an effective mass model to describe electrons moving in a plane of a $2 \mathrm{DEG}$ in a waveguide. The orbital motion couples to the magnetic field component perpendicular to the plane of the 2DEG ( $z$ direction). In $(\mathrm{Cd}, \mathrm{Mn}) \mathrm{Te}$ the exchange interaction between mobile electrons and electrons localized in $\mathrm{Mn}$ atoms gives rise to the giant Zeeman coupling. In the mean-field theory it can be modeled with an effective $g$ factor $g_{\text {eff }}$. We assume that other electron-electron interactions can be omitted for the open conductors considered at typical electron densities and at small magnetic fields. The effective mass Hamiltonian is then

$$
\hat{\mathrm{H}}=\frac{1}{2 m^{*}}[\hat{\mathbf{P}}-\mathrm{eA}(\mathbf{x})]^{2}+\frac{1}{2} g_{\mathrm{eff}} \mu_{\mathrm{B}} \mathbf{B}(\mathbf{x}) \cdot \boldsymbol{\sigma}+V_{\mathrm{dis}}(\mathbf{x}),
$$

where $\hat{\mathbf{P}}$ is the momentum operator, $m^{*}$ is the effective mass, $\mathbf{B}(\mathbf{x})$ is the magnetic field, $\mathbf{A}(\mathbf{x})$ is the vector potential of the $z$ component of $\mathbf{B}(\mathbf{x}), \mu_{\mathrm{B}}$ is the Bohr magneton, $\boldsymbol{\sigma}$ is the vector of Pauli matrices, and $V_{\mathrm{dis}}(\mathbf{x})$ is the scattering potential of the disorder. We assume that the giant Zeeman coupling in $(\mathrm{Cd}, \mathrm{Mn}) \mathrm{Te}$ is the largest spin-dependent energy scale and we omit the Rashba and the Dresselhaus spin-orbit couplings in the Hamiltonian, as discussed above. We use in our calculations the effective mass of CdTe $m^{*}=0.1 m_{e}$, where $m_{e}$ is the bare electron mass. We assume that at low 


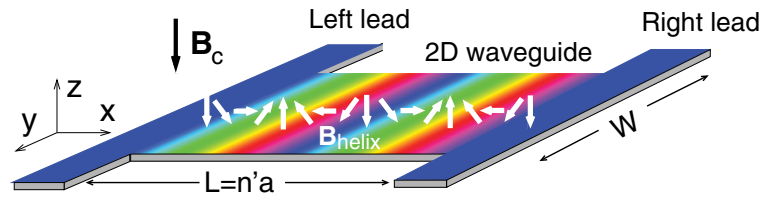

FIG. 1. (Color online) Schematic figure of the system with a 2DEG waveguide of dimensions $L \times W$ and the attached leads. The constant magnetic field $B_{\mathrm{c}}$ is along the $-z$ direction and the helical magnetic field $B_{\text {helix }}$ rotates in the $x z$ plane. The waveguide length $L$ is $n^{\prime}$ helical pitches $a$. In the figure $n^{\prime}=2$ is shown as an example.

temperature the material has a giant Zeeman splitting ${ }^{7}$ with a very large $g_{\text {eff }}$, hence we use here $g_{\text {eff }}$ ranging from 177 to 550 . Disorder is modeled with an Anderson-like impurity model to account for spin-independent scattering processes. ${ }^{8}$

We study both finite rectangular waveguides as well as periodic systems in the direction transverse to the transport direction ( $y$ direction). With periodic boundary conditions we emulate wide systems which would otherwise be beyond computational capabilities. In both cases we calculate magnetoconductance in a domain of length $L$ and width $W$ and the waveguide is connected to leads at $x=0$ and $x=L$. Besides the helical magnetic field we assume a tunable homogeneous magnetic field $\mathbf{B}_{\mathrm{c}}=B_{\mathrm{c}}(0,0,-1)$ perpendicular to the $2 \mathrm{DEG}$ plane. In the leads this gives rise to a significant spin polarization

$$
p=\left(n_{\uparrow}-n_{\downarrow}\right) /\left(n_{\uparrow}+n_{\downarrow}\right),
$$

where $n_{\sigma}$ denotes the number of occupied modes for spin $\sigma=\{\uparrow, \downarrow\}$.

\section{B. Magnetic field texture}

The magnetic field in the calculations has a rotating component which is helical in the transport direction,

$$
\mathbf{B}_{\text {helix }}(\mathbf{x})=-B_{\text {helix }}(\sin 2 \pi x / a, 0, \cos 2 \pi x / a),
$$

where $a$ is the pitch of the helix. The Zeeman energy of the total magnetic field $\mathbf{B}(\mathbf{x})=\mathbf{B}_{\text {helix }}(\mathbf{x})+\mathbf{B}_{\mathrm{c}}$ for parallel $(+)$ and antiparallel (-) spin orientations (for $g_{\text {eff }}>0$ ) is then

$E_{\mathrm{Z}, \pm}(x)= \pm \frac{1}{2} g_{\text {eff }} \mu_{B} B_{\text {helix }} \sqrt{1+\gamma^{2}+2 \gamma \cos (2 \pi x / a)}$,

where $\gamma=B_{\mathrm{c}} / B_{\text {helix }}$. Energies and spin directions of the Zeeman-split bands are depicted in Fig. 2 for different values of $\gamma$. The direction of the total field is

$$
\theta(x)=\arctan \left(\frac{\sin (2 \pi x / a)}{\cos (2 \pi x / a)+\gamma}\right) .
$$

We study spin transmission in the regime where spin transport is predominantly adiabatic..$^{5}$ The magnetic field in the electron's frame of reference then changes slowly on time scales of the order of the period of Larmor spin precession $2 \pi / \omega_{\mathrm{L}}=2 \pi \hbar /\left(g_{\text {eff }} \mu_{B} B\right)$. Denoting the magnetic field modulation frequency in the electron's frame of reference by $\omega_{\text {mod }}=2 \pi v_{\mathrm{F}} / a$ we use $Q=\omega_{\mathrm{L}} / \omega_{\bmod }$ as a measure of the degree of adiabaticity. ${ }^{9} 10$ In ballistic systems the adiabatic regime is $Q \gg 1$. In the presence of disorder the condition is $Q \gg a / l_{e}$, where $l_{e}$ is the electron mean free path. ${ }^{11}$

\section{Landau-Zener model}

In the absence of the homogeneous field component $\theta(x)=$ $2 \pi x / a$, and for electrons moving parallel to the helix axis,

$$
Q_{\text {helix }}=\mu_{B} g_{\text {eff }} B_{\text {helix }} a /\left(2 \pi \hbar v_{\mathrm{F}}\right)
$$

is constant. However, if both field components are present $\theta(x)$ changes faster close to $x=\left(n^{\prime}+\frac{1}{2}\right) a$, where $n^{\prime}$ is an integer [see Fig. 2(b)]:

$$
\frac{\partial \theta(x)}{\partial x}=\frac{\frac{2 \pi}{a}[1+\gamma \cos (2 \pi x / a)]}{1+\gamma^{2}+2 \gamma \cos (2 \pi x / a)} .
$$

The angle $\theta(x)$ is discontinuous at $x=\left(n^{\prime}+\frac{1}{2}\right) a$ in the limit $\gamma \rightarrow 1$, and conditions for adiabaticity are therefore violated. ${ }^{6}$ The Zeeman-split levels are then intertwined and they cross at $x=\left(n^{\prime}+\frac{1}{2}\right) a$ [see Fig. 2(c)]. In this limit a spin wave function which has been transported adiabatically to $x=\left(n^{\prime}+\frac{1}{2}\right) a$ has an overlap of 1 with the upper band with vanishing energy difference between the bands. This means that a transition occurs to the upper band with probability 1 . For spin-polarized states the upper band is at least partially above the Fermi energy and the electron's wave function decays. This leads to spin backscattering. Spin-compensated transport modes are not affected since their energy remains below the Fermi energy. The relative strength of the homogeneous and helical field components therefore determines adiabaticity and the backscattering probability of spin.
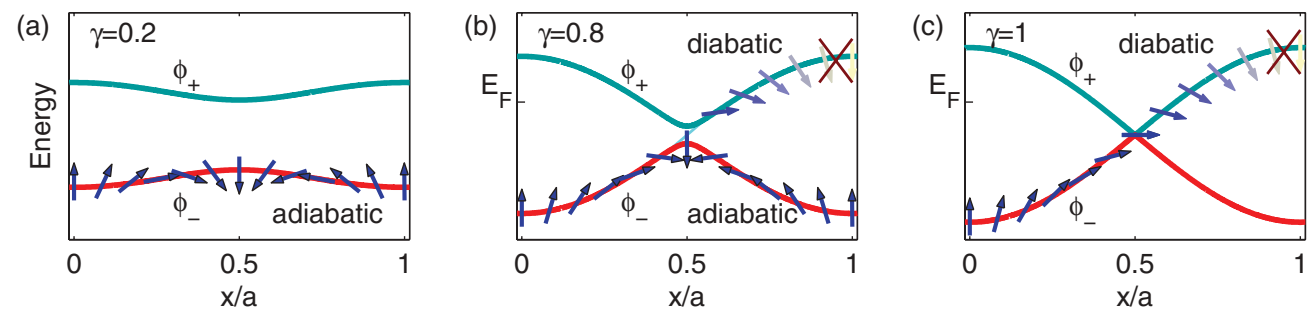

FIG. 2. (Color online) Adiabatic and diabatic spin evolution for a two-level system described by Eqs. (4) and (5) for $B_{\mathrm{c}} / B_{\text {helix }}=\gamma=0.2$ (a), $\gamma=0.8$ (b), and $\gamma=1$ (c). Magnetic field orientation at $x=0$ is down for $g_{\text {eff }}>0$. Fast rotation of the $\phi_{-}$eigenstate spin orientation (arrows attached to the red line) may lead to a diabatic transition to $\phi_{+}$at a level anticrossing (b). If $E_{\mathrm{F}}$ is lower than the energy of $\phi_{+}$the wave function decays and the spin is backscattered at the corresponding potential barrier. At $\gamma=1$ the energy levels cross at $x=a / 2$ (c). Spin which is transported to this point adiabatically in the $\phi_{-}$state has a wave-function overlap of 1 with $\phi_{+}$, and a diabatic transition occurs followed by backscattering. 
The energies of spin-split eigenstates $\phi_{ \pm}$in a combination of homogeneous and helical magnetic fields are given by Eq. (4) and depicted for three representative values of $\gamma$ in Fig. 2. They form a two-level system where diabatic transitions are possible between the states. Landau, Zener, Stückelberg, and Majorana calculated the diabatic transition probability in particular twolevel systems. ${ }^{13-16}$ The levels given by Eq. (4) anticross at $x=\left(n^{\prime}+\frac{1}{2}\right) a$, and a diabatic transition from $\phi_{-}$to $\phi_{+}$occurs with a probability

$$
P=\exp \left(-\frac{2 \pi}{\hbar^{2}} \epsilon_{12}^{2} / \alpha\right),
$$

where the nondiagonal energy term

$$
\epsilon_{12}=\frac{1}{2} \mu_{B} g_{\text {eff }} B_{\text {helix }}|\gamma-1|
$$

equals half the closest distance between the eigenenergies at the closest approach and $\alpha=\frac{1}{\hbar} \frac{d}{d t}\left(\epsilon_{+}-\epsilon_{-}\right)$measures how fast the energies of eigenstates $\phi_{+}$and $\phi_{-}$approach each other during spin transport at the level anticrossing. This depends on the transport velocity. Assuming that an electron moves parallel to the helix axis at speed $v_{\mathrm{F}}$, the energy difference $\epsilon_{+}-\epsilon_{-}$can be approximated from the eigenenergies in the limit $\gamma \rightarrow 1$ yielding

$$
\begin{aligned}
\alpha & =\frac{1}{2} \mu_{B} g^{\text {eff }} B_{\text {helix }} \frac{1}{\hbar} \frac{2 \pi}{a} v_{\mathrm{F}} \lim _{\tau \rightarrow \pi^{-}} \frac{d}{d \tau} \sqrt{2+2 \cos \tau} \\
& =\mu_{B} g^{\text {eff }} B_{\text {helix }} \frac{1}{\hbar} \frac{2 \pi}{a} v_{\mathrm{F}},
\end{aligned}
$$

where $\tau=\frac{2 \pi}{a} v_{\mathrm{F}} t$. In waveguides electrons have a component of momentum perpendicular to the helix axis and effectively $\alpha$ is lower. Using Eqs. (8), (9), and (10) the probability of diabatic transition can be approximated as

$$
P \approx \exp \left[-\pi(\gamma-1)^{2} Q_{\text {helix }} / 2\right],
$$

where $Q_{\text {helix }}$ is given by Eq. (6).

The transition amplitude can also be obtained within the formalism introduced by Dykhne. ${ }^{17,18}$ However, we found that the transition probability in our case of predominantly adiabatic transport does not significantly differ from the Landau-Zener formula (8).

\section{RESULTS}

\section{A. Numerical method}

The magnetoconductance of waveguides with spinpolarized states is calculated using a recursive Green's function (RGF) algorithm based on a tight-binding discretization of the system. ${ }^{12}$ Moreover, we compare the results to the LandauZener approximation for ballistic systems. The electron mean free path $l_{e}$ is estimated from the disorder strength. The transmission coefficients $t_{n m}$ of transport modes are calculated with the RGF algorithm and conductance $G$ is obtained from the Landauer formula

$$
G(B)=G^{0} \sum_{n, m, \sigma, \sigma^{\prime}}\left|t_{n m}^{\sigma \sigma^{\prime}}(B)\right|^{2},
$$

where $G^{0}=e^{2} / h$ is the conductance (per spin) of one channel, $\sigma$ and $\sigma^{\prime}$ denote the spin indices, and $n$ and $m$ are the channel indices. In both leads there is a homogeneous magnetic field
$B_{\mathrm{c}}+B_{\text {helix }}$ perpendicular to the $2 \mathrm{DEG}$ surface. In disordered systems $t_{n m}$ is averaged over random disorder configurations in our calculations. The number of configurations ranges from 10 configurations in large bulklike multimode systems to more than 100000 in single-mode waveguides where the electron mean free path is short. Since the giant Zeeman effect in (Cd,Mn)Te is significant only at low temperatures ( $\mathrm{mK}$ regime) we assume zero temperature in our calculations and do no energy averaging.

\section{B. Tuning of spin backscattering with Landau-Zener transitions}

We first study transitions caused by a single level (anti)crossing in the Zeeman-split bands. Waveguide length is therefore one helical pitch, $L=a$. We omit therefore orbital effects as a first approximation and the effective mass Hamiltonian includes only the kinetic term, the Zeeman coupling, and the disorder potential,

$$
\hat{\mathrm{H}}=\frac{1}{2 m^{*}} \hat{\mathbf{P}}^{2}+\frac{1}{2} g_{\mathrm{eff}} \mu_{\mathrm{B}} \mathbf{B}(\mathbf{x}) \cdot \boldsymbol{\sigma}+V_{\mathrm{dis}}(\mathbf{x}) .
$$

In long waveguides with sequences of level (anti)crossings orbital effects become important and they are studied in Sec. III C. In the case of rectangular waveguides we assume an infinite potential well of width $W$ in the transverse direction. For mode $n$ the quantum well energy is $E_{n}=$ $\left(\hbar^{2} / 2 m^{*}\right)\left(\pi^{2} n^{2} / W^{2}\right)$ for $n=1,2,3, \ldots$ In the simplest case transport involves only one spin-polarized mode $(n=1)$. The spin splitting of this mode, Eq. (4), in the modulated magnetic field gives rise to a two-level system with a periodic sequence of level (anti)crossings (see Fig. 2 for one period).

In ballistic systems the transition probability from the lower spin eigenstate to the higher one can be calculated using either the Landau-Zener approximation, Eq. (11), or the RGF algorithm. For spin-polarized states the upper band is at least partially above the Fermi energy and the wave function decays after a diabatic transition. This leads to spin backscattering. Figure 3 shows the probability of spin backscattering in a single-mode wave function calculated with both methods. In the low $Q_{\text {helix }}$ regime numerical results show a shift in the peak position from $\gamma=1$ towards lower $\gamma$ values [Fig. 3(a)]. Spin transport is not perfectly adiabatic in this regime and spin is slightly nonaligned with the magnetic field resulting in precession. At $\gamma=1$ in the $Q_{\text {helix }} \gg 1$ regime the probability of diabatic transition and spin backreflection tends to 1 [Fig. 2(c)]. The adiabatic theorem is reflected in the probability distribution which gets narrower with increasing $Q_{\text {helix }}$.

The role of spin-independent disorder scattering was analyzed within the RGF formalism. Figure 4(a) shows a dip in the magnetoconductance associated with spin transmission blocking in a disordered single-mode waveguide. We normalize magnetoconductances in the following figures to the corresponding values in a homogeneous magnetic field of strength $B_{\mathrm{c}}+B_{\text {helix }}$ in order to factor out ohmic resistance caused by the disorder. The magnetoconductance calculations at different mean free paths show that almost all transmission is blocked at $B_{\mathrm{c}}=B_{\text {helix }}$ even if the mean free path is shorter than the magnetic field helix pitch $a$ [Fig. 4(b)]. The result can be understood in terms of adiabatic spin transport which remains 

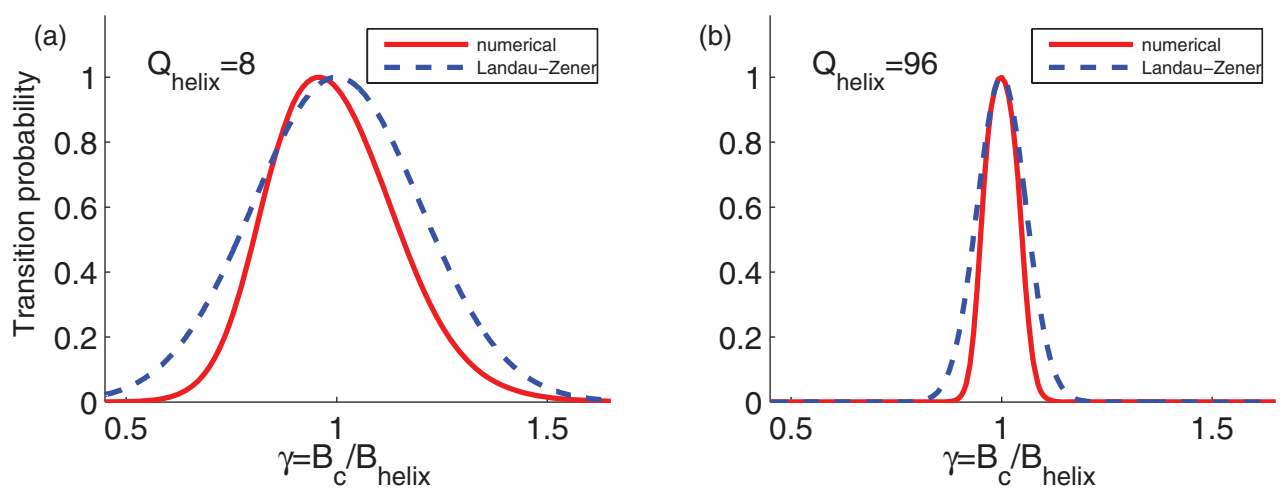

FIG. 3. (Color online) Diabatic transition probability in a ballistic single-mode waveguide of one helical pitch $L=a$ calculated with the RGF algorithm (solid lines) at $a=1.5 \mu \mathrm{m}$ corresponding to $Q_{\text {helix }}=8$ (a) and $a=18 \mu \mathrm{m}$ corresponding to $Q_{\text {helix }}=96$ (b). In the latter case the helical magnetic field changes more slowly in the electron's frame of reference and transport is more adiabatic. Results are compared to the transition probability, Eq. (11), from the Landau-Zener approximation (dashed lines). The adiabaticity parameter $Q_{\text {helix }}$ is approximated using Eq. (6).

spin aligned with the magnetic field despite scattering from disorder. At $\gamma=1$ there is no adiabatic path through the system and spin is reflected [Fig. 2(c)]. With increasing disorder the dip in the relative conductance broadens. Since electrons scatter from impurities they pass the level (anti)crossing many times, which enhances backreflection probability.

The result applies also to multiple spin-polarized channels. Figure 5 shows that in a spin-polarized multichannel system current is almost completely switched off at $\gamma=1$ even in the presence of disorder. There is a small leakage current through the system at $\gamma=1$ because conditions of adiabaticity hold only approximately ( $Q_{\text {helix }} \approx 11$ at $n=1$ and $Q_{\text {helix }} \approx 25$ at $n=8$ in this case) and spin flips are therefore possible. The mean free paths $l_{e}$ and the ratios $l_{e} / a$ in the calculations are of the order of those which are attained in $(\mathrm{Cd}, \mathrm{Mn}) \mathrm{Te}$ quantum wells. $^{6}$

In partially spin-polarized systems Zeeman-split eigenstates $\phi_{ \pm}$of spin-compensated modes both remain below the Fermi energy [see dashed lines in Fig. 6(a)]. Spin backscattering at a level (anti)crossing therefore does not occur and both spins are transmitted in the ballistic case.
This is shown for a double-mode ballistic calculation in Fig. 6(b) where the upper spin-polarized mode is reflected at $B_{\mathrm{c}}=B_{\text {helix }}$ but the lower spin-compensated mode gives $2 G^{0}$ conductance (the normalized relative conductance is therefore $2 / 3)$. Note that if $E_{\mathrm{F}}$ is below the maximum value of the spin-polarized energy band [solid red line in Fig. 6(a)] the wave function of these states decays without a Landau-Zener transition.

In disordered waveguides with partial spin polarization $(p<1)$ the resistance is increased partly due to spin backscattering at Landau-Zener transitions and partly due to disorder scattering, which affects both spin eigenstates. The latter gets more important as disorder increases and there are also transitions between spin-polarized and spin-compensated transport modes. An electron which is initially in a spinpolarized mode may then scatter to another mode which is not backscattered at a level (anti)crossing [e.g., the mode shown with dashed lines in Fig. 6(a)]. As a consequence the electron may transmit and the relative conductance dip at $\gamma=1$ decreases. The total relative conductance depends on the disorder strength as shown in Fig. 6(b).
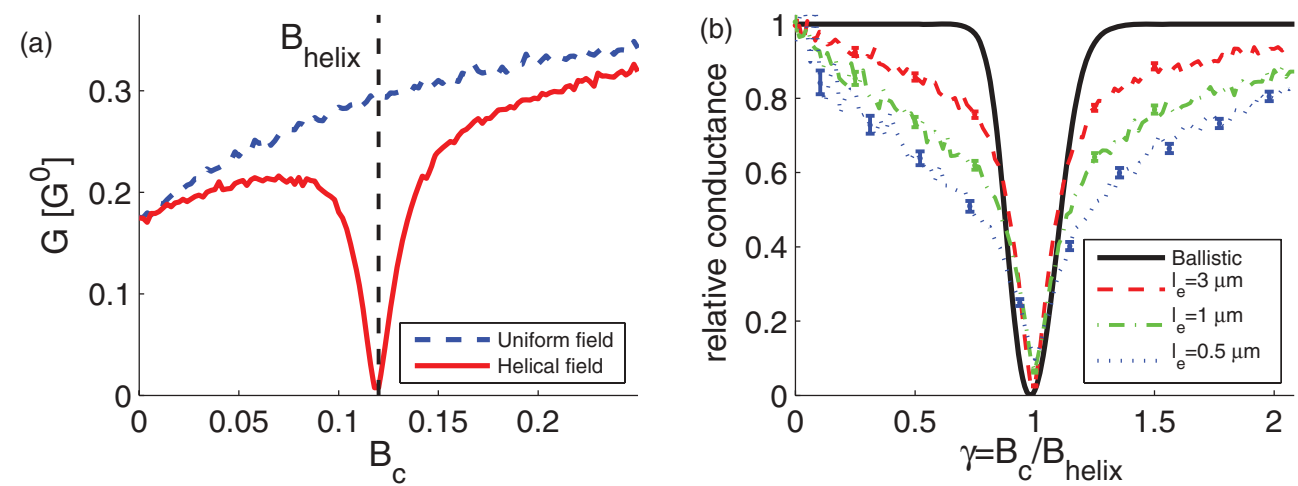

FIG. 4. (Color online) Calculated disorder-averaged transmission in a spin-polarized single-mode waveguide with a helical field of strength $B_{\text {helix }}=0.12 \mathrm{~T}$ and pitch $a=L=1 \mu \mathrm{m}$. Waveguide width is $24 \mathrm{~nm}, m^{*}=0.1 m_{e}, E_{\mathrm{F}}=6.6 \mathrm{meV}$, and $g_{\text {eff }}=177$. (a) Magnetoconductance shows a dip associated with spin backscattering at $B_{\mathrm{c}}=B_{\text {helix }}$. Magnetoconductance in a homogeneous field is shown for comparison. Electron mean free path $l_{e}=3 \mu \mathrm{m}$. (b) Relative magnetoconductance for various electron mean free paths $\left(l_{e}=0.5,1\right.$, and $\left.3 \mu \mathrm{m}\right)$. Conductance is normalized to conductance in a homogeneous magnetic field of the same strength. The energy levels for the ballistic system are shown in Fig. 2. 


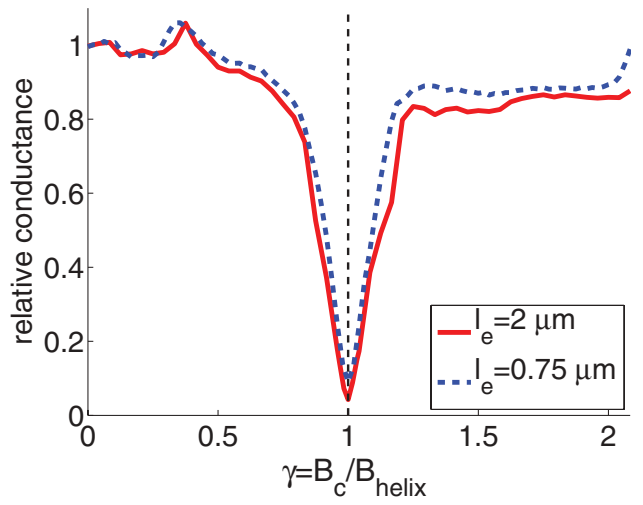

FIG. 5. (Color online) Relative magnetoconductance in a multimode disordered waveguide with eight spin-polarized transport modes at $\gamma=1$. Conductances are normalized to respective conductances in a homogeneous magnetic field of the same strength. Waveguide length is one helical pitch $L=a=1 \mu \mathrm{m}$ and $E_{\mathrm{F}}=$ $8 \mathrm{meV}$. Electron mean free path $l_{e}$ is indicated in the figure.

The above results are directly applicable to bulklike multimode systems. Figure 7 shows magnetoconductance in a

(a)
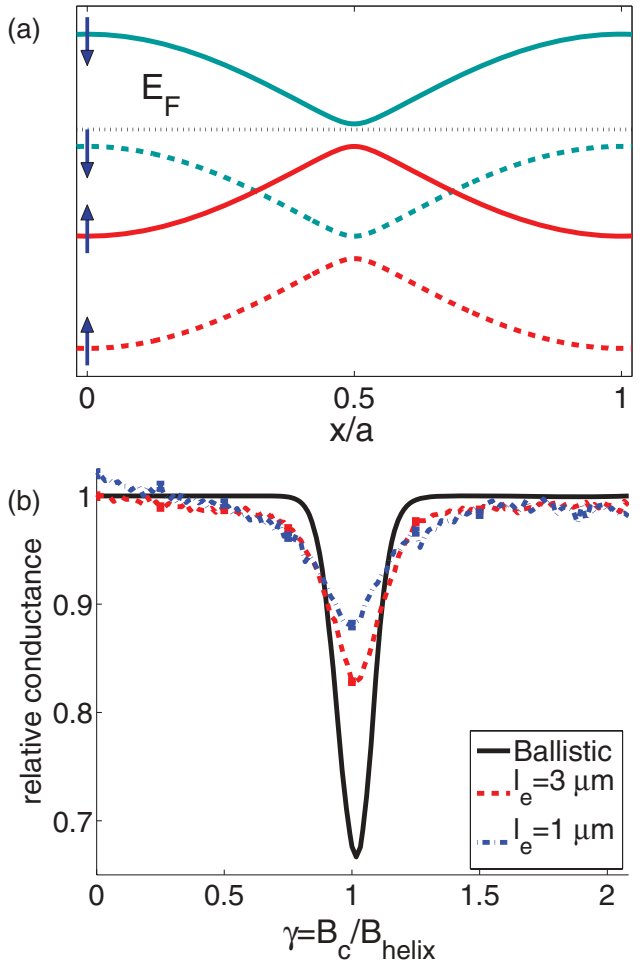

FIG. 6. (Color online) (a) In a multimode waveguide energies of the Zeeman-split eigenstate pairs are either both below the Fermi energy (dashed lines) giving rise to a spin-compensated mode, or the higher Zeeman-split eigenstate (solid lines) is above the Fermi energy $E_{\mathrm{F}}$ giving rise to a spin-polarized mode. Eigenstates are plotted at $\gamma=0.8$. The arrows show the corresponding spin directions at $x=0$. (b) Relative magnetoconductance calculated in a double-mode ballistic waveguide (solid line) and disordered waveguides (dashed line for $l_{e}=3 \mu \mathrm{m}$ and dash-dotted line for $l_{e}=1 \mu \mathrm{m}$ ). The energy levels of the modes are depicted in (a); the lower mode is spin compensated and the upper mode is spin polarized, $p=0.33$. The system parameters are otherwise the same as in the caption of Fig. 5.

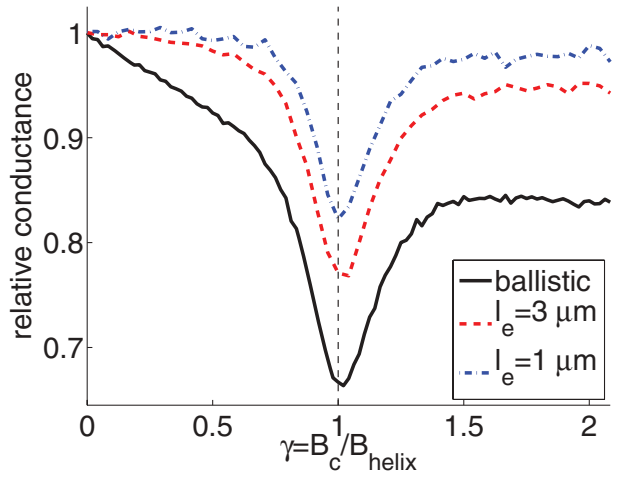

FIG. 7. (Color online) Relative magnetoconductance in a partially polarized $(p=0.34$ at $\gamma=1)$ disordered waveguide with 170 transverse modes. Waveguide length $L=a=1 \mu \mathrm{m}$ and width is $3 \mu \mathrm{m}$. Conductance is normalized to conductance in a homogeneous magnetic field of the same strength. $E_{\mathrm{F}}=6.6 \mathrm{meV}$ and $g_{\text {eff }}=$ 550 .

partially polarized multimode system $(n=170)$ where a similar conductance pattern develops due to spin backscattering. The magnetoconductance is asymmetric with respect to $\gamma=1$ since the calculations are done at constant $B_{\text {helix }}$ and therefore spin polarization of the leads increases with $\gamma$.

Although an electron may scatter at impurities, the spin still aligns with the local external magnetic field if it changes slowly in the electron's rest frame $(Q \gg 1)$. In the diabatic transport regime $(Q \ll 1)$ the spin wave function becomes a superposition of local eigenstates which leads to spin precession in the local magnetic field. The abovedescribed way to control spin transmission is then not possible.

\section{Sequences of level (anti)crossings}

A single (anti)crossing in the Zeeman-split energy levels has a spin transmission blocking effect as shown in Sec. III B. This causes an increase in resistance which depends on spin polarization and disorder strength. Resistance modulation is enhanced if electrons are transported through a sequence of level (anti)crossings ( $n^{\prime}$ helical modulations, $L=n^{\prime} a$ ). The electron transmission probability depends on transitions between transport channels caused by disorder scattering or orbital dynamics in the magnetic field. Hence we take orbital effects into account and use Hamiltonian (1) to calculate magnetoconductance with the RGF method.

In ballistic systems there are no transitions between transport channels in the adiabatic transport limit since the local transverse modes change slowly with the magnetic field. However, if the local magnetic field in the electron's frame of reference changes rapidly, transitions between the modes may lead to reoccupation of a backscattered spinpolarized mode [e.g., in Fig. 6(a) these transitions would be from the spin-compensated modes (dashed lines) to spinpolarized modes (solid lines)]. The electron may subsequently backscatter in the following level (anti)crossing. The relative magnetoconductance therefore decreases with magnetic field helix pitch at $\gamma=1$ [see Fig. 8(a)]. Neither a pure Zeeman coupling nor orbital coupling alone account for the clear-cut 
(a)

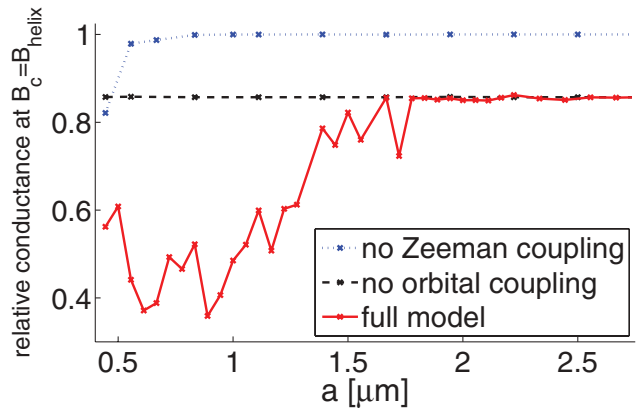

(b)

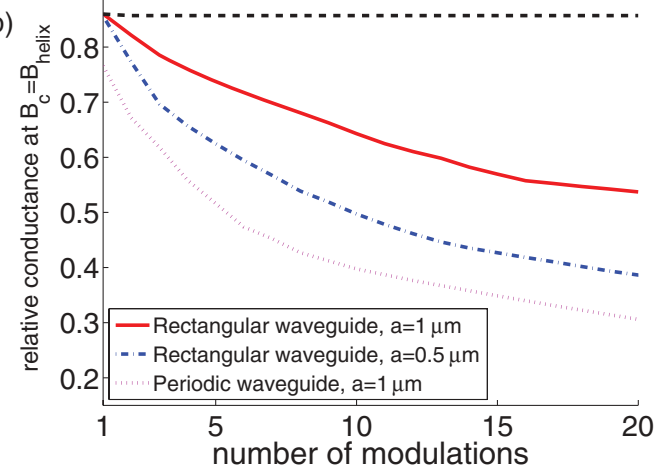

FIG. 8. (Color online) Relative magnetoconductance in long ballistic waveguides with $n^{\prime}$ helical modulations. Magnetoconductance is calculated at $B_{\mathrm{c}}=B_{\text {helix }}=0.12 \mathrm{~T}, g_{\text {eff }}=177$, and $p=0.14$. (a) Relative magnetoconductance as a function of the helix pitch $a$ in the case $n^{\prime}=16$. The figure shows relative magnetoconductance calculated with the full model Hamiltonian [Eq. (1), solid line], in the absence of the Zeeman coupling ( $g_{\text {eff }}=0$, dotted line), and in the absence of the orbital coupling $(\mathbf{A}=\mathbf{0}$, dashed line $)$. Adiabaticity parameter $Q_{\text {helix }} \approx 1$ for the mode $n=1$ at $a=0.5 \mu \mathrm{m}$. Waveguide width is $W=425 \mathrm{~nm}$, number of transport modes $n=16$, and $E_{\mathrm{F}}=7.4 \mathrm{meV}$. (b) Relative magnetoconductance as a function of $n^{\prime}$ in a rectangular waveguide (for $a=1 \mu \mathrm{m}$, solid line, and for $a=0.5 \mu \mathrm{m}$, dash-dotted line) and in a periodic system in the $y$ direction for $a=1 \mu \mathrm{m}$. Full model Hamiltonian is used here. The dashed line shows the relative magnetoconductance in the adiabatic limit. The waveguide width $W=350 \mathrm{~nm}$.

reduction in the relative conductance for $a \leqslant 1.5 \mu \mathrm{m}$. For magnetoconductance traces as a function of magnetic field in the ballistic case see Supplemental Material in Ref. 6.

Figure 8(b) shows magnetoconductance in a partially polarized ballistic waveguide as a function of the number of helical modulations $n^{\prime}$ in the Zeeman-split energy bands. Conductances are calculated at $\gamma=1$ where the diabatic transition probability is highest. The degree of adiabaticity is lower in the short helix pitch $a=0.5 \mu \mathrm{m}$ and the probability of mode transitions is higher. The relative conductance decrease is amplified with increasing $n^{\prime}$ and results in a huge dip in magnetoconductance at $\gamma=1$ if the number of modulations is large. We find qualitatively similar but quantitatively larger effects in periodic systems [dotted line in Fig. 8(b)].

The above mechanism causes enhanced spin blocking also in disordered waveguides. The resistance is effectively higher for the spin-polarized channels than for spin-compensated channels due to diabatic transitions and spin backscattering. The dip in the relative magnetoconductance at $\gamma=1$ increases

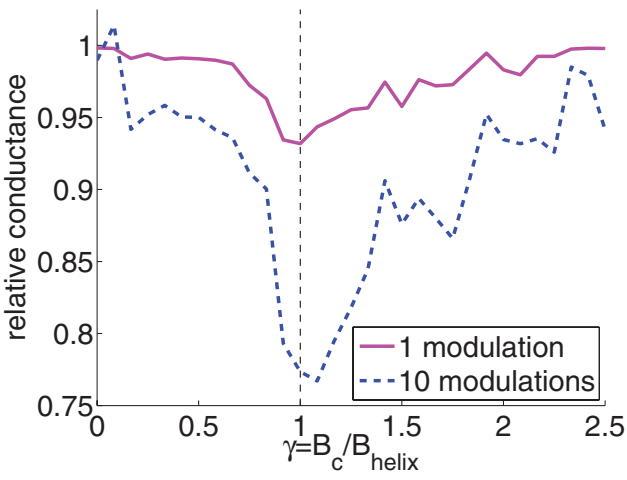

FIG. 9. (Color online) Relative magnetoconductance in a partially spin-polarized $(p=0.11)$ disordered waveguide for one helical modulation (solid line) and for ten modulations (dashed line). Magnetic field helix pitch is $a=0.5 \mu \mathrm{m}$ in both cases and $Q_{\text {helix }} \approx 1$ for the mode $n=1$. Electron mean free path $l_{e}=3 \mu \mathrm{m}$, waveguide width $W=350 \mathrm{~nm}, g_{\text {eff }}=177, E_{\mathrm{F}}=6.6 \mathrm{meV}, B_{\text {helix }}=0.12 \mathrm{~T}$, and the number of transport modes is 16 . The calculation is done at zero temperature. The smaller peaks at $\gamma>1$ reflect the particular choice of energy and geometry of the system.

with the number of magnetic modulations (Fig. 9). This is in line with the experiments in Ref. 6. Figure 10 shows the relative conductance at $\gamma=1$ in disordered waveguides with spin polarization $p=0.11$. We note that the relative conductance change at $n^{\prime}=15$ for $l_{e}=1 \mu \mathrm{m}$ is larger than the relative conductance change at $n^{\prime}=1$ in the ballistic case.

\section{CONCLUSIONS AND OUTLOOK}

Our results show that spin transistor action can be realized via tunable Landau-Zener transitions. The mechanism is tolerant against spin-independent disorder scattering for an Anderson impurity model. Completely spin-polarized systems show full spin backscattering, and thus current switching, even when the mean free path of electrons is of the order of the magnetic modulation length.

In partially spin-polarized waveguides the resistance modulation decreases with increasing disorder strength. However,

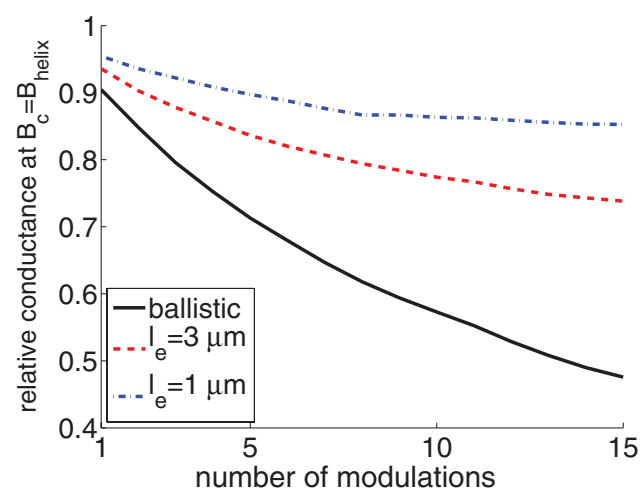

FIG. 10. (Color online) Relative magnetoconductance in a partially spin-polarized $(p=0.11)$ disordered waveguide at $\gamma=1$ as a function of the number of magnetic modulations (in helix pitches $a=0.5 \mu \mathrm{m})$. The waveguide parameters are otherwise the same as in Fig. 8(b). 
the resistance modulation due to Landau-Zener transitions can be enhanced with a sequence of (anti)crossings in the spin-split bands. Orbital transitions ca use successive reoccupation and backscattering of spin-polarized modes. This effect also provides an explanation as towhy the spin-blocking effect in experiments is larger than the theoretical prediction for ballistic systems in the absence of orbital effects. ${ }^{6}$

Implementation of a spin transistor mechanism via tunable Landau-Zener transitions might be a more feasible approach to realize spin transistor functionality than controlling spin dephasing times using an interplay of Rashba and Dresselhaus spin-orbit couplings. ${ }^{19}$ In the latter proposal the transistor operation is based on the persistent spin helix state ${ }^{20}$ which is also tolerant against spin-independent disorder scattering. However, device operation requires a delicate adjustment of the spin-orbit parameters. Moreover, the spin splitting is bounded by the Dresselhaus spin-orbit coupling strength that depends on the crystal lattice structure.

Several technical challenges remain before our concept can be realized in a useful spin transistor device. The magnetic fields for spin transmission control could be generated with magnetic gates (see Supplemental Material in Ref. 6). The giant Zeeman effect in known materials is significant only at low temperatures. Nevertheless, the presented spin-blocking mechanism can be applied also for other spin-splitting interactions which persist to higher temperatures. For a more thorough discussion of the device development aspects we refer to Ref. 6. Our concepts may also be applied to other materials where helical spin ordering is present, such as the interface of multiferroic oxides. ${ }^{21}$ To conclude, robustness of the spin-blocking effect via tunable Landau-Zener transitions provides a promising alternative strategy to design spin transistor functionality with enhanced efficiency and disorder tolerance.

\section{ACKNOWLEDGMENTS}

We thank M. Wimmer for providing the code for the recursive Green's function transport equation solver, V. Krueckl for help with implementing the periodic boundary conditions, C. Betthausen for careful reading of the manuscript and comments, and D. Weiss for many helpful discussions. We acknowledge financial support from the Deutsche Forschungsgemeinschaft through SFB 689 (H.S., K.R.) and Elitenetzwerk Bayern (T.D.).

\footnotetext{
"henri.saarikoski@gmail.com

${ }^{1}$ Semiconductor Spintronics and Quantum Computation, edited by

D. D. Awschalom, D. Loss, and N. Samarth (Springer, Berlin, Heidelberg, 2009).

${ }^{2}$ S. Datta and B. Das, Appl. Phys. Lett. 56, 665 (1990).

${ }^{3}$ H. C. Koo, J. H. Kwon, J. Eom, J. Chang, S. H. Han, and M. Johnson, Science 325, 1515 (2009).

${ }^{4}$ I. Žutić, J. Fabian, and S. Das Sarma, Rev. Mod. Phys. 76, 323 (2004).

${ }^{5}$ M. Born and V. Fock, Z. Phys. A Hadrons and Nuclei 51, 165 (1928).

${ }^{6}$ C. Betthausen, T. Dollinger, H. Saarikoski, V. Kolkovsky,

G. Karczewski, T. Wojtowicz, K. Richter, and D. Weiss, Science 337, 324 (2012).

${ }^{7}$ J. K. Furdyna, J. Appl. Phys. 64, R29 (1988).

${ }^{8}$ T. Ando, Phys. Rev. B 44, 8017 (1991).

${ }^{9}$ M. V. Berry, Proc. R. Soc. London, Ser. A 392, 45 (1984).
}

${ }^{10}$ D. Frustaglia, M. Hentschel, and K. Richter, Phys. Rev. Lett. 87, 256602 (2001).

${ }^{11}$ M. Popp, D. Frustaglia, and K. Richter, Phys. Rev. B 68, 041303(R) (2003).

${ }^{12}$ M. Wimmer and K. Richter, J. Comput. Phys. 228, 8548 (2009).

${ }^{13}$ L. D. Landau, Phys. Z. Sowjetunion 2, 46 (1932).

${ }^{14}$ C. Zener, Proc. R. Soc. London, Ser. A 137, 696 (1932).

${ }^{15}$ E. C. G. Stückelberg, Helvetica Physica Acta 5, 369 (1932).

${ }^{16}$ E. Majorana, Nuovo Cimento 9, 43 (1932).

${ }^{17}$ A. M. Dykhne, Sov. Phys. JETP 14, 941 (1962).

${ }^{18}$ J. P. Davis and P. Pechukas, J. Chem. Phys. 64, 3129 (1976).

${ }^{19}$ J. Schliemann, J. C. Egues, and D. Loss, Phys. Rev. Lett. 90, 146801 (2003).

${ }^{20}$ B. A. Bernevig, J. Orenstein, and S.-C. Zhang, Phys. Rev. Lett. 97, 236601 (2006).

${ }^{21}$ C. Jia and J. Berakdar, Phys. Rev. B 81, 052406 (2010). 